\title{
Empregando Redes Bayesianas para modelar automaticamente o conhecimento dos aprendizes em Lógica de Programação
}

\author{
Using Bayesian Networks to model learner's knowledge in Programming Logic
}

Juliano Vier, João Gluz, Patricia A. Jaques

Programa Interdisciplinar de Pós-graduação em Computação (PIPCA)

Universidade do Vale do Rio dos Sinos (UNISINOS)

juliano.vier@yahoo.com.br, jcgluz@unisinos.br, pjaques@unisinos.br

\begin{abstract}
Resumo Sistemas Tutores Inteligentes (STI) são ambientes computacionais que possuem a capacidade de aprender sobre os aprendizes e modelar esse conhecimento em um Modelo de Aluno, de forma que possam prover assistência individualizada. Uma das técnicas inteligentes utilizadas para modelar o conhecimento do aluno são as Redes Bayesianas. Trata-se de modelos probabilísticos utilizados para cálculo de probabilidades de eventos relacionados. Este artigo apresenta um Modelo do Aluno probabilístico no domínio de lógica de programação que possui representações para os conceitos especificos desta área. A construção da Rede Bayesiana foi realizada com o auxílio de especialistas da área em questão. Para avaliação do modelo criado, foi empregada a técnica de alunos virtuais. Os resultados da avaliação evidenciam a viabilidade do modelo proposto.
\end{abstract}

Palavras-Chave: Sistemas Tutores Inteligentes. Modelo de Aluno. Redes Bayesianas. Ensino de Programação.

\begin{abstract}
Intelligent Tutoring Systems (ITS) are computing environments that have the ability to learn about students, so that they can provide personalized assistance. One of the techniques used to model student's knowledge is the Bayesian Networks. These networks are probabilistic models used to calculate the probabilities of related events. This work presents a probabilistic Student Model on programming logic. The construction of the Bayesian network was made with the assistance of experts, and the evaluation of the model was performed with the use of virtual students. The evaluation results show evidences of the viability of the proposed model.
\end{abstract}

Keywords: Intelligent Tutoring Systems. Student Model. Bayesian Networks. Programming Teaching. 


\section{Introdução}

As disciplinas de programação têm o objetivo de ensinar conceitos de lógica de programação e a sintaxe das linguagens de programação. Para que a aprendizagem de programação seja efetiva, a realização de exercícios é tão importante quanto a instrução dada em aula. A prática de exercícios de programação está relacionada à estratégia de Resolução de Problemas (RP, Problem solving, em inglês). Em cursos de programação, a resolução de problemas geralmente envolve fornecer aos estudantes a descrição de um problema, que deve ser quebrado em subproblemas menores, implementados usando linguagem e ambiente de programação especificados pela ementa da disciplina, testados e novamente integrados para a solução do problema original [23]. RP está incluída de forma implícita ou explícita na maioria das disciplinas introdutórias de programação.

Normalmente, os exercícios (problemas) de programação são desafiadores para os alunos. Estudantes que enfrentam dificuldades buscam junto ao professor um melhor entendimento dos seus erros e também a melhor forma de procurar o conhecimento necessário para criar uma implementação (programa) capaz de resolver o problema proposto.

Entretanto, os professores muitas vezes demoram em fornecer um feedback apropriado sobre os exercícios, devido à demanda excessiva. Essa atraso no retorno pode ser frustrante aos aprendizes. Além disso, do ponto de vista do professor, realizar a correção de todos os trabalhos de programação pode se tornar uma tarefa extenuante e longa, se a demanda for representativa. Nesse caso, muitas vezes, torna-se difícil fornecer um atendimento adequado aos alunos em termos de tempo e detalhamento da correção.

Ao invés da correção repetitiva de exercícios, os docentes poderiam empregar o seu tempo para auxiliar os alunos de forma mais efetiva, por exemplo, fornecendo auxílio aos aprendizes que apresentam maior dificuldade na compreensão e entendimento dos conteúdos. Nesse caso, um professor, com maior disponibilidade de tempo, pode atender essa demanda de forma muito mais satisfatória, tendo os alunos assim um auxílio mais personalizado.

Nesse sentido, software educacionais podem ser empregados para auxiliar o professor na correção automática e no auxílio daqueles casos mais comuns de dúvidas dos alunos. Em especial, os Sistemas Tutores Inteligentes (STI) são ambientes computacionais inteligentes que podem ser bastante úteis neste contexto. Esses sistemas empregam Inteligência Artificial para modelar o conhecimento do aluno e prover diversos tipos de assistência individualizada aos estudantes, entre os quais, correção em tempo real, passo-a-passo dos exercícios, ajuda, etc. $[39,49]$.

Um dos principais componentes de um Sistema Tutor Inteligente é o Modelo do Aluno [9, 10, 37]. Este módulo é responsável por garantir o ensino personalizado, ou seja, devidamente adaptado às necessidades individuais do aprendiz. Isto é possível, pois este módulo infere e armazena de forma persistente informações sobre o aluno em questão, tais como seu grau de conhecimento, estados afetivos, motivação, etc, o que permite ao STI adaptar seu modo de ensino para o aprendiz [49].

Uma das técnicas empregadas para inferir e modelar o conhecimento do aluno são as Redes Bayesianas [19, 35]. Estas são modelos probabilísticos através dos quais se pode obter informações sobre a probabilidade de certos eventos acontecerem [24, 29]. Este trabalho propõe um Modelo de Aluno probabilístico, implementado como uma Rede Bayesiana, que fornece informações importantes sobre o domínio do aluno em conceitos específicos (também chamados de "unidades de conhecimento" no domínio de STI [45]) para um Sistema Tutor Inteligente, voltado ao ensino de programação. Espera-se, no futuro, oferecer aos alunos deste sistema, com as informações disponibilizadas pelo modelo, um feedback muito mais preciso e adequado.

Para a construção desta rede, especialistas da área, professores com experiência no ensino de programação, foram consultados. A partir das entrevistas realizadas, foi possível chegar a um modelo representativo do domínio, onde os conceitos se inter-relacionam pela relação de prérequisito. Para verificar os resultados finais apresentados por este modelo, foi utilizada a abordagem de alunos virtuais.

Este artigo encontra-se organizado como segue. A seção 2, apresenta os principais conceitos envolvendo Redes Bayesianas. Na seção 3, é abordado o ensino de programação. Na Seção 4, são apresentadas as principais noções sobre Sistemas Tutores Inteligentes. Os trabalhos Relacionados são discutidos na seção 5. O Modelo de Aluno desenvolvido é apresentado na seção 6 e a sua avaliação na seção 7. Finalmente, na seção 8 , são apresentadas as conclusões e algumas ideias de trabalhos futuros.

\section{Redes Bayesianas}

Redes Bayesianas (RB) são modelos probabilísticos que permitem lidar de forma rigorosa com a representação de conhecimentos em domínios onde existe incerteza $[25,28,29]$. As RB oferecem duas vantagens importantes para modelar este tipo de conhecimentos: (i) permitem uma representação gráfica das relações causais entre as variáveis do domínio, mas que leva em conta o grau ou 
peso da relação causal através de probabilidades [29] e (ii) oferecem um mecanismo eficiente e exato de cálculo da distribuição de probabilidades das variáveis do domínio [27].

As RB se apresentam na forma de um grafo acíclico direcionado, onde cada um dos nós representa uma variável do domínio, ou seja, um evento específico com estados mutuamente exclusivos que pode ocorrer no domínio e que tem importância no contexto da modelagem. As arestas representam as causas e/ou influências que um nó tem sobre outros nós. Cada nó possui uma tabela de probabilidades anexada que indica as probabilidades do evento em questão acontecer. Se o evento não for dependente de nenhum outro evento (ou seja, não existe nenhuma relação ou aresta direcionada de algum outro nó para o nó que representa o evento), então a tabela de probabilidades representa as probabilidades a priori ou marginais para o evento e é denominada de Tabela de Probabilidades Prévia. Se, por outro lado, houver uma ou mais relações causais (arestas) de outros eventos (nós) para um evento (nó), então a tabela de probabilidades irá definir as probabilidade condicionais associadas ao evento, sendo chamada de Tabela de Probabilidade Condicional do nó. Os nós com arcos dirigidos para o nó que representa o evento, são denominados de nós pais.

A Figura 1 representa uma Rede Bayesiana em um cenário proposto por [34]. Neste cenário, temos uma residência com um alarme contra roubos que ocasionalmente dispara de maneira equívoca quando um terremoto acontece. O proprietário da residência tem dois vizinhos, João e Maria, que estão incumbidos de ligar para o mesmo caso o alarme venha a disparar. Temos cinco eventos diferentes (Roubo, Terremoto, Alarme, JoaoLiga e MariaLiga) que estão representados pelos nós da rede, sendo que cada nó tem uma Tabela de Probabilidade Condicional.

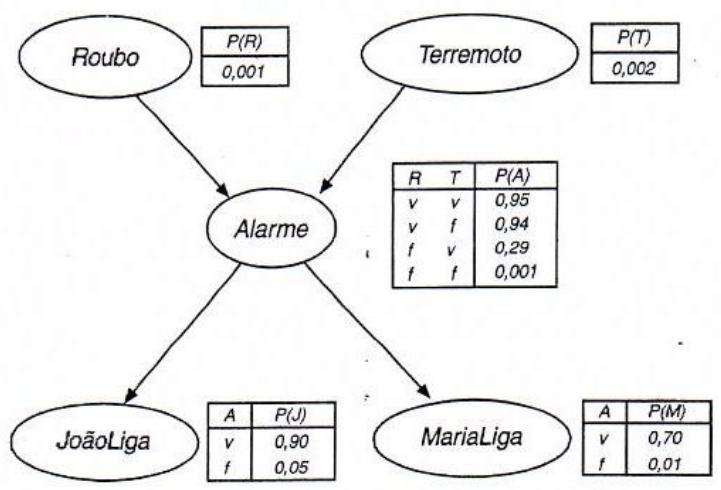

Figura 1: Exemplo de Rede Bayesiana

A probabilidade de um evento acontecer é uma informação importante em uma Rede Bayesiana. Cada evento pode assumir dois ou mais estados, e cada estado tem uma probabilidade de acontecer. Estes estados são mutuamente exclusivos e exaustivos, ou seja, apenas um por vez poderá acontecer, ao mesmo tempo em que ao menos um deles deverá acontecer. A soma das probabilidades dos eventos de um estado deve ser 1. A informação a respeito das probabilidades pode ser obtida de várias formas [24]:

- Pelo princípio da indiferença (todos os eventos são equiprováveis, se não existe razão para acreditar no contrário);

- Através de frequências relativas, ou observação de um evento por tempo suficiente para que seja possível calcular a probabilidade deste acontecer (a proporção entre o total de observações e o número de vezes em que determinado evento foi observado);

- Utilizando um grau de confiança, ou probabilidade subjetiva, que é a crença que um indivíduo tem de que certo evento pode acontecer. Essa técnica é particularmente útil quando as probabilidades são fornecidas por especialistas para eventos que não podem ser observados através de frequências relativas.

Por exemplo, a probabilidade de um arremesso de dado resultar com o valor 'seis' é $1 / 6$, de acordo com o princípio da indiferença. Entretanto, se forem observados suficientes arremessos de dados, é possível perceber que a probabilidade de obter o valor 'seis' é próxima de $1 / 6$ (pode-se supor a observação de 600 arremessos e com isso encontrar 98 vezes o valor 'seis' durante o experimento).

Por outro lado, o resultado de uma partida de futebol não pode ser estimado utilizando-se destes métodos. Portanto, o máximo que podemos conseguir é uma probabilidade subjetiva, que representa o quanto um indivíduo acredita que o evento pode acontecer, ou seja, é uma crença pessoal e varia de acordo com quem estiver estimando.

\subsection{Probabilidade Condicional}

Define-se a probabilidade de que um evento ' $A$ ' tenha um resultado ' $a$ ' como $\mathrm{P}(\mathrm{A}=\mathrm{a})$. Se este evento for $\mathrm{o}$ arremesso de um dado, por exemplo, pode-se definir então da seguinte maneira: $\mathrm{P}(\mathrm{A}=$ seis $)=1 / 6$, ou $\mathrm{P}($ seis $)=$ $1 / 6$, como sendo a probabilidade de se obter um 'seis como resultado. Se este evento for a retirada de uma carta do baralho pode-se definir como $\mathrm{P}$ (rei_de_copas) $=1 / 52$, para a probabilidade de se obter um 'rei_ $\overline{d e}$ copas' como a carta retirada.

Probabilidade Condicional diz respeito ao quão provável um evento é de acontecer, dado o acontecimento de outro evento. No caso da retirada de uma carta do bara- 
lho, se existe o conhecimento prévio de que esta carta é um 'rei', a probabilidade da carta ser um 'rei_de_copas' é $1 / 4$, pois é possível encontrar apenas quatro reis no baralho, e um deles será o de copas. Define-se da seguinte forma: $\mathrm{P}($ rei_de_copas $\mid$ rei $)=1 / 4$.

A fórmula para o cálculo da Probabilidade Condicional é a seguinte [34]:

$P(a \mid b)=P\left(a^{\wedge} b\right) / P(b)$

Que também pode ser reescrita da seguinte forma (denominada Regra do Produto):

$P\left(a^{\wedge} b\right)=P(a \mid b) P(b)$ 'b'.

Onde $\mathrm{P}\left(\mathrm{a}^{\wedge} \mathrm{b}\right)$ é a simultaneidade dos eventos ' $\mathrm{a}$ ' $\mathrm{e}$

\subsection{Independência}

Existem dois tipos de Independência: a absoluta e a condicional. Ambas são importantes na construção de Redes Bayesianas. Esta importância existe devido à quantidade de eventos, e assim a necessidade de construir uma tabela de probabilidades, chamada de Distribuição Conjunta Total (DCT). Esta tabela mostra a probabilidade de todos os eventos atômicos do domínio que está sendo observado.

A Distribuição Conjunta Total lista todas as probabilidades possíveis, e a partir dela é possível identificar as probabilidades condicionais existentes no domínio, bem como relacionamentos de independência. O problema é que o tamanho desta tabela tem crescimento exponencial em relação ao número de eventos ou variáveis distintas de um dado domínio. Mesmo em domínios que não possui muitas variáveis, por exemplo 30 ou 40 (que é o caso do presente trabalho), torna-se impraticável fazer inferências probabilísticas por meio da DCT.

A tabela 1 foi retirada de [34]. Ela mostra todas as probabilidades para o conjunto de variáveis (eventos) booleanas 'DorDeDente', 'Carie' e 'Boticao', através de uma Distribuição Conjunta Total.

Através desta Distribuição Conjunta Total é possível descobrir, por exemplo, que a probabilidade de um indivíduo ter 'carie' e 'dordedente', mas não ser necessário o uso de 'boticao' é igual a:

$P\left(\right.$ carie $^{\wedge}$ dordedente ${ }^{\wedge} \sim$ boticao $)=0,012$

Contudo, muitas vezes, o domínio é composto de dezenas ou até mesmo centenas de variáveis, fazendo com que a tabela se torne extensa, e impossível de se computar na prática. Isso ocorre porque a tabela correspondente à DCT tem uma complexidade de memória exponencial em relação à quantidade de variáveis do modelo.
Tabela 1: Distribuição conjunta total.

\begin{tabular}{|lllll|}
\hline \multicolumn{3}{c}{ dordedente } & \multicolumn{2}{l|}{$\sim$ dordedente } \\
\hline & boticao & $\sim$ boticao & boticao & $\sim$ boticao \\
carie & 0,108 & 0,012 & 0,072 & 0,008 \\
$\sim$ carie & 0,016 & 0,064 & 0,144 & 0,576 \\
\hline
\end{tabular}

Por esta razão, a percepção de independências é muito importante. No caso da tabela anterior, se fosse inserida mais uma variável 'Tempo', esta não booleana e com quatro valores possíveis, então a DCT, que possuía oito entradas, terá com esta nova inclusão 32 entradas. Isso ilustra a complexidade inerente a esse tipo de organização de dados, e por que a utilização desta tabela acaba se tornando inviável quando se tem muitas variáveis.

Nesse caso, entretanto, podemos questionar o nível de relação entre as variáveis. Russel e Norvig [34] colocam que a probabilidade de 'Tempo' é completamente independente das outras variáveis, pois as condições climáticas não interferem (ou sofrem interferências) em uma dor de dente ou mesmo a utilização de um boticão. Logo, a relação entre estas probabilidades é:

$P\left(\right.$ dordedente ${ }^{\wedge}$ carie $^{\wedge}$ boticao ${ }^{\wedge}$ Tempo $=$ nublado $)=$ $P($ Tempo $=$ nublado $) P\left(\right.$ dordedente ${ }^{\wedge}$ carie $^{\wedge}$ boticao $)$ (4)

Ou seja, existe uma relação de independência absoluta entre 'Tempo' e as outras variáveis. Sendo assim, não existe a necessidade de adicionar esta variável na tabela de Distribuição Conjunta Total anterior, pois estaria repleta de informações redundantes. Pode-se então criar duas tabelas: a anterior, contendo a distribuição de 'dordedente', 'carie' e 'boticao', e outra com as probabilidades a priori (ou seja, não condicionais) de 'Tempo', que terá apenas quatro entradas.

Então a independência absoluta entre duas variáveis ' $a$ ' e ' $b$ ' pode ser escrita como:

$P(a \mid b)=P(a)$ ou $P(b \mid a)=P(b)$

$\mathrm{Ou}$ :

$P\left(a^{\wedge} b\right)=P(a) P(b)$

Além da independência absoluta vista com a inserção da variável 'Tempo', existe a independência condicional. Neste exemplo, Russel e Norvig [34] colocam que 'DorDeDente' e 'Boticao' são independentes dada a presença de 'Carie'. Isso pode ser percebido da seguinte forma:

$P($ DorDeDente, Boticao $\mid$ Carie $)=$ P(DorDente | Carie) P(Boticao | Carie)

Isso acontece, pois 'DorDeDente' não tem relação direta com 'Boticao', mas ambos possuem relação direta com 'Carie' (pode-se dizer que 'Carie' é a causadora de 
ambas). A fórmula a seguir determina a independência condicional entre duas variáveis ' $a$ ' $\mathrm{e}$ ' $b$ ', dado o conhecimento prévio de ' $c$ ':

$$
P\left(a^{\wedge} b \mid c\right)=P(a \mid c) P(b \mid c)
$$

Com o conhecimento das independências entre as variáveis do domínio, pode-se fragmentar a tabela original em tabelas menores. Essa vantagem percebe-se mais claramente quando da construção das redes Bayesianas, na próxima subseção.

\subsection{Teorema de Bayes}

Nem sempre se tem o conhecimento de todas as entradas da tabela de DCT. Isso acontece em situações onde existe o conhecimento prévio de alguns eventos, mas não de todo o domínio [42]. Por exemplo, um médico pode descobrir a probabilidade de um paciente ter uma doença específica baseado em algum sintoma. Se este médico sabe a probabilidade deste sintoma aparecer, dada a presença da doença em questão, e também conhece as probabilidades a priori de um indivíduo apresentar este sintoma e de um indivíduo estar acometido de tal doença, ele pode utilizar o Teorema de Bayes.

O Teorema de Bayes permite o cálculo de uma probabilidade condicional dado que se tenha a probabilidade condicional no sentido inverso e também a probabilidade a priori de ambos os eventos envolvidos na probabilidade condicional. Dados dois eventos ' $a$ ' e ' $b$ ', sendo que $\mathrm{P}(\mathrm{b})$ é diferente de zero, a fórmula para efetuar este cálculo é [24, 29]:

$$
P(a \mid b)=\frac{P(b \mid a) P(a)}{P(b)}
$$

\subsection{Inferência Bayesiana}

A Rede Bayesiana (RB) é uma representação completa do domínio que opera de maneira semelhante a uma Distribuição Conjunta. Na verdade, a RB representa todos os relacionamentos de independência e dependência condicional nitidamente, de forma que tais relacionamentos estejam muito mais visíveis do que em uma tabela.

Assim, a tabela DCT que teria um número muito grande de entradas para definir todas as probabilidades do domínio em questão, pode usualmente ser representada por uma Rede Bayesiana mais compacta e com um modelo gráfico mais compreensível. Para chegar a esse ponto, é necessário identificar todos (ou pelo menos a maioria) os relacionamentos de dependência e independência condicional e isto corresponde a uma tarefa não trivial.

$\mathrm{Na}$ Figura 1, a topologia da rede exprime exatamente as relações de independência percebidas pelo criador da mesma. Percebe-se que roubos e terremotos afetam diretamente a probabilidade do alarme soar, ao mesmo tempo em que João e Maria somente ligam quando ouvem o alarme, ou seja, o fato de um dos dois ligar é independente de outros fatores. Neste caso, eles não verificam se realmente está ocorrendo um roubo ou um terremoto, eles apenas ligam para o proprietário quando ouvem o alarme. Além disso, nem João nem Maria ligam sempre que ouvem o alarme, pois podem estar distraídos, ou absortos em alguma atividade ou mesmo estarem ausentes.

Em resumo, uma $\mathrm{RB}$, se construída adequadamente, nada mais é do que uma representação fiel da tabela de Distribuição Conjunta Total do domínio sendo modelado. Dessa forma, assim como através da tabela é possível calcular quaisquer probabilidades conjuntas de eventos (variáveis) do domínio, através da RB os mesmos cálculos podem ser efetuados com muito mais eficiência [27], além do benefício da clareza e de uma melhor percepção do domínio [34].

\section{Ensino de Programação}

A aprendizagem de uma linguagem de programação é um dos primeiros passos percorridos no caminho daqueles que decidiram seguir os cursos de graduação da área de Informática (por exemplo, Ciência da Computação e Sistemas de Informação). Com este aprendizado estarão aptos a aprender muitos outros assuntos pertinentes à sua futura formação. Mas, além destes, existem também muitos alunos de outros cursos que se aventuram por cursos de programação, por conta da necessidade de saber noções básicas sobre o assunto.

Entretanto, tanto para o primeiro grupo quanto para o segundo, a programação se apresenta como matéria repleta de detalhes e desafios. Segundo Rogalski e Samurcay [33], trata-se realmente de tarefa complexa com dificuldades relevantes, de modo que somente com algum tipo de tutoria adequada é que os resultados poderão ser atingidos em tempo razoável e de forma satisfatória.

As disciplinas de programação têm o objetivo de ensinar aspectos básicos sobre a lógica de programação e a sintaxe das linguagens de programação. Para isto acontecer, é importante que o aluno pratique a programação. Um professor define projetos de programação específicos que devem ser implementados em aula ou extraclasse, e através destas atividades os alunos colocarão em prática conceitos importantes vistos em aula de maneira teórica. Após, os estudantes devem, então, enviar seus programas, em forma de código fonte, para que o professor avalie se a implementação está correta. Em termos práticos, esta é uma estratégia didático-pedagógica, chamada de resolução de problemas [23], muito empregada para este domínio de ensino.

Existem muitas questões que são decisivas na escolha do currículo, e muitas estão relacionadas com a lingua- 
gem escolhida [30]. Um exemplo é o fato de a linguagem ser orientada a objetos, pois este paradigma determinará, por exemplo, uma metodologia de ensino diferenciada. Também é possível que um currículo priorize alguns conceitos específicos, de tal maneira que estes sejam vistos de forma mais evidente durante o curso.

Além do currículo, aspectos pedagógicos também devem ser levados em consideração, pois estes tratam de questões relativas a como o conhecimento será ensinado/aprendido [30]. Estes aspectos são importantes a fim de garantir e facilitar o aprendizado desejado. Neste sentido, cabe frisar que, seguindo as tendências, o ensino de programação também sofreu mudanças significativas na forma de ensinar, passando do modelo centrado no instrutor pra o modelo centrado no estudante.

Pears et al. [30] discorrem sobre três ênfases comuns utilizadas no ensino de programação:

* Capacidade de resolver problemas: muitos educadores resumem a programação como sendo a aplicação de determinadas habilidades a fim de solucionar problemas específicos. Quando um aluno aprende a resolver um determinado problema, ele pode utilizar este conhecimento na resolução de outros problemas similares. Se o problema for muito parecido, o conhecimento será utilizado plenamente; mas se o problema for apenas levemente semelhante, além do conhecimento, $\mathrm{o}$ aluno terá que utilizar outras habilidades;

* Aprendizagem de uma linguagem de programação: uma das visões mais tradicionais no ensino de programação é a que prioriza aspectos específicos da linguagem ensinada. Nesse caso, o foco são a sintaxe e as estruturas da linguagem em questão, por exemplo, o ensino de uma linguagem orientada a objetos, onde aspectos específicos desta abordagem são enfatizados durante o processo de aprendizagem;

* Produção de código: quando o foco são os programas criados pelo aluno, ou seja, a codificação de aplicativos é a prioridade, a ênfase é dada ao resultado dessa codificação. Entretanto, nesse caso é necessário definir com clareza se a abordagem de ensino será em torno de uma programação mais básica ou mais avançada.

De qualquer forma, independente de metodologia e pedagogia utilizadas, a aprendizagem de programação implica em esforço e dedicação por parte do estudante, e outros tipos de ferramentas que possam auxiliar durante este processo são sempre bem-vindas.

\section{Sistemas Tutores Inteligentes}

Sistemas Tutores Inteligentes (STI), em inglês Intelligent Tutoring Systems (ITS), tem o propósito de auxiliar o aluno na aprendizagem dos mais variados temas. Várias técnicas de Inteligência Artificial (IA) e conceitos de ciência cognitiva são integrados em uma aplicação que tem como intuito ensinar, ou ao menos assistir neste processo, de forma personalizada. Um STI é um ambiente computacional que objetiva fornecer condições de aprendizagem de forma objetiva e adaptativa, além de feedback, para alunos de uma disciplina específica $[37,38$, 40, 49].

O STI é na verdade uma evolução natural das ferramentas CAI (Computer-Aided Instruction), que é um termo utilizado para designar a utilização do computador na educação. Com o avanço da IA e a ampliação do poder de processamento das máquinas, foi possível adicionar inteligência a estas ferramentas, e consequentemente adaptar a estratégia de ensino utilizada de forma a adequar-se ao aluno.

Além das questões já citadas, Woolf [49] argumenta que as rápidas mudanças nos campos da educação, ciência cognitiva e inteligência artificial são responsáveis por gerar uma verdadeira reviravolta no mecanismo dos processos educacionais, fazendo com que novas tecnologias tomem um lugar de destaque no meio acadêmico. Neste contexto, os STI visam auxiliar os estudantes de maneira individualizada e adaptativa.

A respeito da IA, estudos tem mostrado que sistemas educacionais que fazem uso de mais recursos desta área são geralmente mais efetivos a nível educacional [41]. Isso ocorre porque as diversas técnicas de IA empregadas permitem inferir mais informações sobre o aluno e fornecer uma assistência mais individualizada.

De fato, estudos demonstram que estudantes aprendem mais efetivamente na modalidade professor particular (one to one tutoring) do que em uma sala de aula com vários alunos. Segundo Bloom [8], os aprendizes que receberam ensino individualizado tiveram, em média, um desempenho, na ordem de 2 sigmas (desvio-padrão), melhor que os alunos que receberam instrução convencional (um professor para uma sala de aula). Algumas revisões sistemáticas recentes mostraram que STIs podem ser tão eficientes quanto professores particulares [21, 43, $44,46]$. E essa efetividade é melhor observada em sistemas tutores do tipo step-based, que são ainda mais adaptativos por fornecer assistência personalizada em cada passo do problema resolvido pelo aluno [46].

Apesar da comprovada efetividade dos STIs, tem havido certa resistência no uso destas ferramentas [45]. Isto se deve, principalmente, a uma ideia equivocada de que o 
STI venha a substituir o professor, principalmente devido ao termo 'tutor', que tem conotação autoritária e pode transmitir a ideia da substituição. Porém, o objetivo do STI não é necessariamente esse, e sim apoiar o ensino. Sobre essa questão, na verdade são as políticas pedagógicas do desenvolvedor e também do professor que vão definir o papel do STI.

\subsection{Modelo do Aluno}

O STI precisa ter meios de inferir e representar o conhecimento adquirido pelo aluno, bem como raciocinar sobre este conhecimento, a fim de atender melhor às necessidades individuais dos estudantes. Além do conhecimento do estudante no domínio, alguns STIs também modelam outras informações dos estudantes, tais como emoções, estilos de aprendizagem, traços de personalidade, motivação, etc. [2, 3, 50]. O conhecimento do aluno, bem como essas informações individuais, pode ser obtido através de questionários, assim como da observação do comportamento do estudante no STI. Esta é a tarefa do Modelo do Aluno: monitorar e analisar as atividades do estudante, e inferir e armazenar informações que possam representar de forma adequada características relevantes do mesmo $[37,38]$.

Quanto mais informações o tutor possuir a respeito do aluno, mais preciso será o modelo. Entretanto, é importante organizar as informações de forma coerente. STIs armazenam no modelo do aluno informações que eles presumem que representem com maior fidelidade o conhecimento do estudante sobre o domínio.

O conhecimento do estudante é inferido pelo STI durante a execução das tarefas, pois é durante a resolução de problemas que o aluno demonstra o seu conhecimento ao sistema. Os dados da resolução do estudante são as evidências de conhecimento que o STI possui sobre o aprendiz [45]. Esses dados são empregados pelo modelo de inferência do tutor para verificar que habilidades foram exercitadas pelo estudante e seu nível de conhecimento em cada habilidade. Na literatura, é muito comum o uso de Redes Bayesinas para inferir o nível de conhecimento do estudante nas habilidades envolvidas no domínio $[13,22]$, já que está é uma poderosa de ferramenta de inferência para dados incertos (ver seção 2).

A presença de um Modelo do Aluno em um STI e a utilização deste para adaptar o tutor às necessidades do estudante proporciona um ganho de desempenho para o aluno de pelo menos $10 \%$ [40]. Portanto, além da adição de um Modelo de Aluno, se faz necessário que o tutor tenha plenas condições de utilizá-lo, com isto garantindo uma melhora no processo de ensino ou assistência ao aprendiz.

O Modelo do Aluno fornece dados (informações) para outros módulos do STI, e de forma mais evidente para o módulo tutor. Este poderá utilizar estas informações para melhor adequar a ferramenta às necessidades do estudante. Pode-se dizer que o propósito do Modelo de Aluno é fornecer informações que serão usadas a fim de proporcionar feedback personalizado, e também orientar o aluno de forma individualizada durante o curso. Isso porque pessoas diferentes aprendem em ritmos diferentes e de maneiras diferentes [48].

Apesar dos STIs disponíveis apresentarem diversas diferenças entre si, a questão envolvendo a modelagem do estudante é invariavelmente a mais importante [20]. E isto é esperado, afinal o estudante é a parte mais importante, sendo o objetivo final do sistema em si fazer com que o aluno aprenda o conhecimento pretendido.

\section{Trabalhos Relacionados}

Redes Bayesianas têm sido amplamente empregadas para a modelagem do conhecimento do aluno em STIs voltados aos mais diversos domínios $[4,15,36]$. O processo de modelagem da RB depende do domínio, pois ao conceber a rede bayesiana é necessário definir quais são os nós da rede (geralmente, os conceitos envolvidos) e as relações causais entre os nós (geralmente, relação de prérequisitos entre os conceitos). Nesta seção são descritos trabalhos relacionados que igualmente empregam redes bayesianas com o objetivo de modelar o conhecimento do estudante.

Em [26], redes bayesianas são utilizadas em um STI onde o objetivo é o ensino de estruturas de dados (array, lista encadeada, pilha e árvore). Neste trabalho, os autores consideram que conhecer um conceito mais específico possui uma influência causal no fato de conhecer um conceito mais genérico, de forma que a rede pode ser facilmente visualizada como 'agrupamentos' de conceitos relacionados entre si. As evidências são inseridas na rede através de nós específicos para este fim (alimentados com o resultado de um exercício/teste), e recebem influência dos nós considerados necessários para uma resposta correta. Nesse trabalho, não é possível visualizar relações entre diferentes conteúdos de forma macro, já que os conceitos trabalhados (estruturas de dados) podem ser aprendidos em qualquer ordem (independentemente).

Andes é um STI cujo objetivo principal é auxiliar no ensino introdutório de física. A ferramenta provê feedback imediato e auxílio específico para o aluno [17]. Um Modelo do Aluno com Rede Bayesiana é utilizado para determinar as próximas ações do tutor, que mudam de acordo com o progresso do estudante. Enquanto este estiver resolvendo os problemas de maneira correta, o tutor simplesmente indica que o caminho está correto. A Rede Bayesiana tenta descobrir qual estratégia o aluno está tentando utilizar para encontrar a solução, através de múltiplos caminhos conhecidos possíveis, que são gera- 
dos pelo tutor. Através de comparações, Andes tenta equiparar uma das soluções já conhecidas com a solução que o estudante está apresentando [49]. Nesse caso, o modelo de aluno gera uma rede distinta para cada problema sendo solucionado, o que implica em alguma adaptação na rede bayesiana quando um novo exercício for adicionado no STI. No presente trabalho, como a rede obtém como evidência o conceito empregado pelo estudante, não há necessidade de remodelagem da rede.

Em [11], um tutor para o ensino de programação também é proposto. Apesar de escolher a linguagem $\mathrm{C}++$, esse tutor também tem como foco a programação para estudantes que estejam iniciando a programação. Entretanto, a abordagem utilizada para a construção da rede foi apenas a análise curricular de um curso específico da linguagem utilizada. Além disso, para definir as probabilidades, foi utilizado apenas um cálculo simples feito em cima de alguns dados estatísticos, método este considerado discutível por [14]. O modelo foi inserido em um STI chamado BITS. Este sistema apresenta ao aluno explicações sobre certos conceitos, e, após a leitura, pergunta ao estudante se o mesmo entendeu o assunto, e baseado nesta resposta, atualiza o modelo do aluno. Para avaliar o modelo, foi utilizada uma abordagem com alunos virtuais, semelhante em alguns aspectos com a utilizada por $[12,47]$, com a diferença de que neste trabalho os alunos virtuais não são modelados como um subconjunto de regras do sistema especialista. Diferente da análise curricular realizada em [11], o modelo de aluno bayesiano proposto neste artigo foi baseado em uma detalhada análise do conhecimento de vários especialistas (professores) no ensino de programação, que possuem experiência acumulada no ensino desta disciplina.

\section{Modelo Bayesiano de Aluno para Ensino de Programação}

Como explicado anteriormente, o presente trabalho propõe um modelo de aluno implementado com Rede Bayesiana para o domínio de lógica de programação. Essa rede será utilizada por um STI para prover assistência individualizada aos aprendizes. Para que isso seja possível, a rede precisa inferir, a partir das evidências, o conhecimento do estudante. As evidências serão nós específicos da rede, que representam o resultado de exercícios/testes resolvidos pelo aprendiz.

\subsection{Definição dos nós e relações causais da Rede Bayesiana}

A construção de uma disciplina para um curso de programação pode envolver um grande número de pessoas e as decisões podem ser questionáveis [30]. Para este traba- lho, levantou-se uma coleção de conceitos para serem ensinados em um curso desta natureza. Esta seleção foi fortemente influenciada pela ementa das disciplinas introdutórias de programação de uma universidade comunitária no sul do Brasil (Programação I/Laboratório I), mas também incluiu conceitos vistos na disciplina subsequente (Programação II/Laboratório II).

De modo geral, esse fator foi determinante para que a linguagem escolhida fosse a mesma ministrada nestas disciplinas (Java), assim como o fato de os professores entrevistados (especialistas) serem docentes destas. Soma-se o fato de que a linguagem Java está entre as mais evidentes no contexto atual da tecnologia (apresentandose como uma das mais relevantes no momento) e a abundância de ferramentas e cursos disponíveis para a mesma $[6,7]$.

Além da seleção da linguagem, a confecção da Rede Bayesiana apresentou desafios relevantes. Em um primeiro momento, um conjunto pequeno e primário de conceitos foi selecionado a partir de trabalhos onde o currículo de cursos de programação foi fragmentado de forma conveniente $[1,31]$. Essa fragmentação permitiu elencar as primeiras variáveis que constituíram a rede, sendo que este modelo inicial permaneceu presente e representativo, ainda que tenha sofrido algumas mudanças significativas, como inclusão de vários outros nós e reposicionamento de alguns outros.

As primeiras impressões acerca deste conjunto inicial de variáveis não eram conclusivas e muito menos esclarecedoras, de modo que definir as relações entre os nodos não foi tarefa trivial. Visualizar quais conteúdos seriam pré-requisitos para outros conteúdos constitui um desafio considerável mesmo para especialistas.

Entretanto, verificando publicações de nível didático, como $[16,18]$, foi possível visualizar alguns conceitos elementares do domínio. Dessa forma, alguns conceitos puderam ser classificados como pré-requisitos de outros, ainda que neste momento esta classificação não tenha sido suficientemente extensa e precisa.

Após a rede ter sido contemplada com as relações entre as variáveis (estabelecidas até este momento com base na análise curricular e em publicações [16, 18]), fez-se necessária a participação de especialistas do domínio em questão. Neste caso, três professores de programação foram consultados. Todos ministram disciplinas introdutórias do curso de Ciência da Computação da universidade cujo objetivo é o ensino de programação em linguagem Java.

O primeiro especialista entrevistado é Mestre em Informática e tem 40 anos de experiência com ensino de programação de várias linguagens, sendo as principais: Fortran, Cobol, Basic, Pascal e Java. O segundo é Mestre 
em Computação e ensina programação faz 36 anos. Já ministrou aulas de Basic, Fortran, Pascal e Java, entre outras. E, por fim, o terceiro professor que colaborou com seu conhecimento especializado é Mestre em Ciência da Computação e tem 5 anos de experiência ensinando as linguagens Java e C.

Cada professor foi consultado individualmente em duas oportunidades diferentes, sendo que cada reunião teve tempo de duração entre 30 e 45 minutos. Após a primeira reunião com todos os professores, a rede inicial montada a partir do currículo da disciplina foi ajustada de acordo com as considerações destes. Em um segundo momento, os especialistas puderam observar o resultado de suas considerações iniciais, e assim avaliar melhor a influência das mesmas no modelo final. Desta vez, algumas poucas alterações foram sugeridas, com baixo impacto na estrutura da rede.

Aos especialistas foi dada a liberdade para que opinassem sobre a estrutura da rede, de modo que visualizassem as relações existentes como pré-requisitos e assim pudessem contribuir para a expansão da mesma. Os especialistas sugeriram inúmeras correções e vários acréscimos. Este procedimento foi importante para a estabilização da Rede Bayesiana que é o cerne deste trabalho. Somente com o conhecimento do especialista, refinando o modelo inicial, é que foi possível aproximar-se de uma rede que pudesse espelhar com fidelidade razoável as relações entre os conteúdos.

Cabe ressaltar que a seleção dos conteúdos contemplados com presença na rede não tem o intuito de apresentar-se como completa e/ou definitiva, mas sim suficientemente relevante para o trabalho em questão. É fato que alguns conceitos mais avançados (relacionados a estruturas de dados, tais como árvores binárias e grafos) foram omitidos. No entanto, o intuito foi povoar a rede com aqueles conteúdos considerados essenciais em cursos de programação de nível introdutório. Entre estes, estão principalmente aqueles encontrados em disciplinas de primeiro semestre nos cursos de computação.

A relação de causalidade entre os nós da rede foi utilizada como forma de definir os pré-requisitos entre os conteúdos [20]. Desta forma, a influência que um nó exerce sobre outro na verdade indica uma relação onde o nó que influencia é pré-requisito de aprendizagem do nó influenciado (neste caso onde os nós representam conceitos/conteúdos).

Sobre a relação do tipo pré-requisito, é preciso dizer que se trata de uma relação de necessidade, mas não chega a significar uma relação de suficiência. Isto quer dizer que os pré-requisitos são necessários para o entendimento de um conteúdo, mas o simples fato de conhecêlos não é certeza de que o conteúdo é conhecido.

\subsection{Definição das probabilidades}

Após a definição de todos os nós que seriam parte integrante e definitiva da rede em questão, iniciou-se o processo de definição das probabilidades. Este procedimento, feito com acompanhamento dos especialistas, tentou definir, entre os pré-requisitos de cada conteúdo, aqueles essenciais e também os mais relevantes.

A definição de probabilidades é um dos fatores mais delicados do processo, pois lida diretamente com o fator incerteza [14]. As questões relativas à incerteza são as que justificam o uso de uma Rede Bayesiana no modelo de aluno, mas também são o ponto crucial da criação da rede.

Devido a certos fenômenos conhecidos como slips (equívocos ou deslizes) e lucky guesses ('chutes' ou 'adivinhações') [20, 32], os valores percentuais utilizados na rede nunca foram considerados absolutos. Isto é, não podemos assumir um completo desconhecimento do aluno (caso ele cometa um erro), pois em determinado momento ele pode ter tido um lapso ou cometido um simples deslize (slip). Por outro lado, um conhecimento absoluto também é radical (caso ele acerte uma questão), pois o estudante pode ter arriscado uma resposta, tendo obtido sorte na 'adivinhação' do resultado (lucky guess).

Existem ainda aqueles estudantes que respondem erradamente de forma proposital, apenas para verificar qual será o comportamento do sistema [32] ou para tentar obter a resposta sem esforço, o que é conhecido como Gaming The System [5]. Por essas razões, é difícil determinar com exatidão absoluta o nível de conhecimento do aluno, de forma que é razoável assumir uma margem de erro.

De uma forma geral, sendo $\mathrm{X}$ a variável de evidência que define que o aluno conhece um conceito $\mathrm{Y}$, então a probabilidade de um slip pode ser dada por:

$P(\sim X \mid Y)$

Assim como a probabilidade de um lucky guess será dada por:

$P(X \mid \sim Y)$

Além das exceções acima explicadas, existem inúmeras dificuldades inerentes ao processo de atribuir percentuais aos nós da rede. No caso do presente trabalho, estes valores dizem respeito à porcentagem de chances que um aluno teria de conhecer determinado conceito, dado que este conheça (ou não) todos (ou alguns) de seus prérequisitos.

No caso dos nós (conceitos) com apenas um prérequisito, este valor foi arbitrariamente definido como 99\%, devido a chance de um lucky guess. Para conceitos com vários pré-requisitos, fez-se necessária uma análise 
mais precisa, a fim de definir quais das relações são imprescindíveis e quais deveriam receber um peso maior nos valores percentuais.

Esta análise baseou-se em alguns fatores: se um prérequisito é considerado essencial para a aprendizagem de um conceito, então o desconhecimento deste elimina as chances de conhecer este conceito. Da mesma forma, quando os pré-requisitos não são considerados essenciais, foi necessário descobrir, entre estes, quais seriam aqueles com mais influência para que o aluno pudesse conhecer o conceito em questão.

Desta forma, as tabelas de probabilidades condicionais para todos os nós foram preenchidas, sempre buscando valores razoáveis e coerentes com a realidade.

\subsection{Estrutura da rede}

Em seu estado final, a Rede Bayesiana construída para este trabalho conta com 45 nós, excluindo-se os nós utilizados para entrada de evidências. Além destes, a rede foi contemplada com 80 arestas, representando todas as relações encontradas. Entretanto, até chegar a este resultado, a rede passou por várias melhorias e sofreu certo número de acréscimos e decréscimos. A estrutura final da rede pode ser vista na Figura 2.

Para construção, modelagem e testes, foi utilizado o aplicativo UnBBayes (http://unbbayes.sourceforge.net/), versão 3.52.7. O UnBBayes foi desenvolvido em Java e oferece uma interface gráfica onde a rede pode ser desenhada facilmente, além de permitir visualizar a propagação das evidências por toda a rede, quando algum dos nós é evidenciado.

Antes de chegar à rede final, muitos valores foram testados, e vários nós foram removidos por várias razões: seja porque eram dispensáveis do ponto de vista da programação introdutória, seja para reduzir a complexidade da rede, ou ainda para evitar redundâncias desnecessárias.

Todos os nós presentes na rede representam conceitos que seriam encontrados em um curso de programação para iniciantes, ou seja, aspectos básicos e elementares da disciplina, como por exemplo: variável, operadores aritméticos, laços de repetição, expressões condicionais e objetos. Entretanto, alguns conceitos um pouco mais avançados também foram tratados na rede, com o intuito de enriquecê-la e também devido ao fato de estarem intimamente ligados a outros conceitos, como: arrays multidimensionais, exceções e recursão.

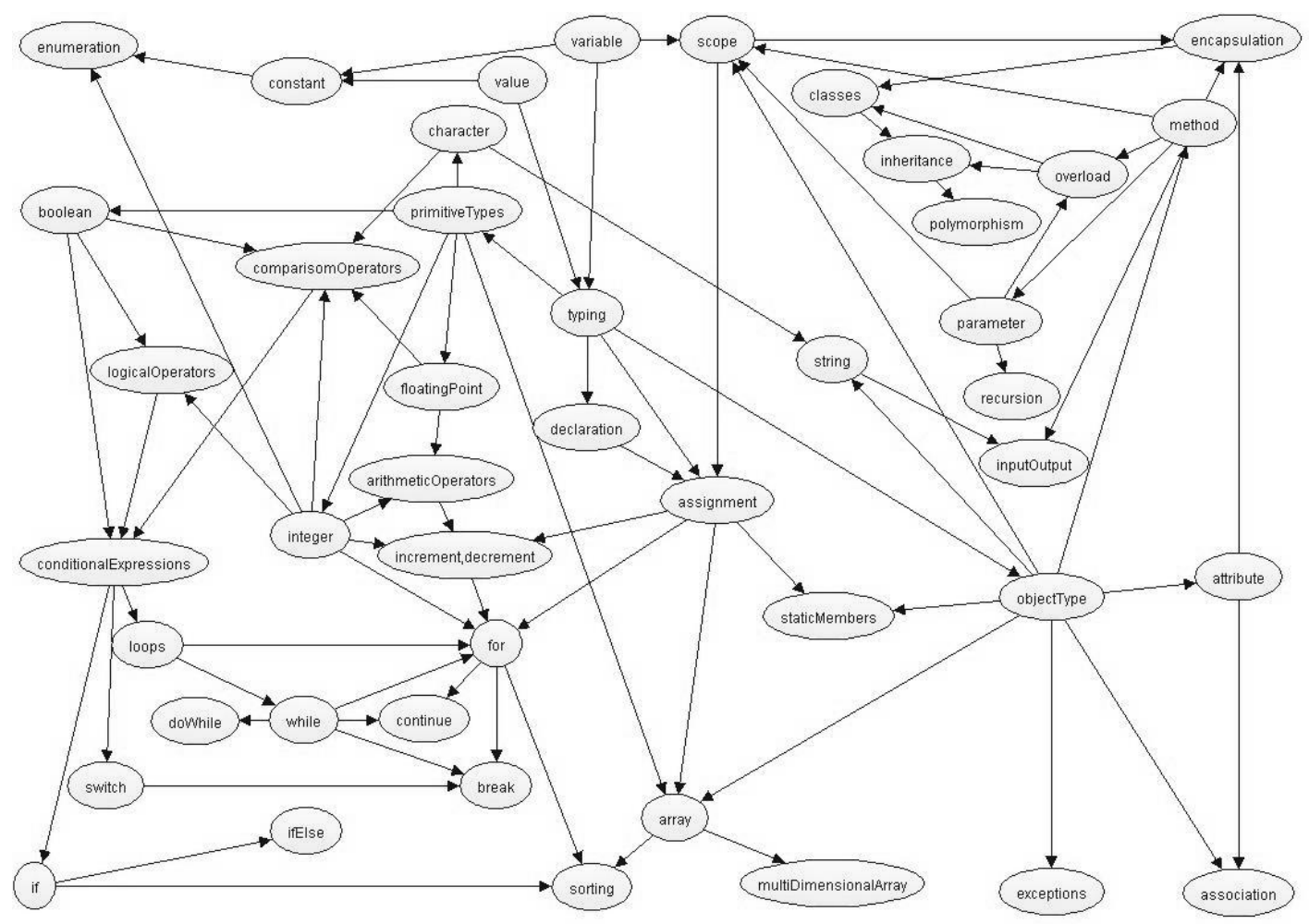

Figura 2: Estrutura da Rede Bayesiana Proposta 
Dado o número de variáveis (nós) presente na rede, e a quantidade de relações entre elas, o desenho desta adquiriu a partir de certo momento uma complexidade relevante. A rede perdeu a característica de árvore (mais visível no início da implementação) e adquiriu um visual de grafo, como pode ser observado na Figura 2.

Alguns nós possuem apenas uma relação básica: nós que são apenas pré-requisitos para outros nós (variável e valor) e nós que não são pré-requisitos para nenhum outro (associação, polimorfismo, if-else). Além destes, existem aqueles que se encontram no centro da rede, e cujas relações podem ser simples, ou seja, recebem influência de apenas um nó e influenciam apenas um outro nó (atributos e switch-case). Outros nós possuem relações complexas, como o caso do conceito for, que tem cinco pré-requisitos e também age como pré-requisito de outros três conceitos.

\subsection{Entrada de evidências}

A rede apresenta uma grande estrutura de nós que representam conhecimentos que o aluno pode possuir. Esses conhecimentos são conceitos de um curso de programação. Entretanto, fez-se necessária a inclusão de alguns nós cuja função é a de receber evidências do conhecimento do aluno (Figura 3). Este nó representa o resultado de um teste/exercício específico a qual o aluno tenha sido submetido. Um esquema semelhante foi utilizado em [26].

Este nós recebem a evidência de aprendizado do aluno: se ele acertou o exercício/teste, então o nó receberá um valor de $100 \%$ de certeza, caso contrário o nó receberá um valor de $0 \%$. Neste modelo, quanto mais evidências forem incluídas na rede, maior será a precisão com que se pode aferir o conhecimento do aluno sobre os vários conceitos.

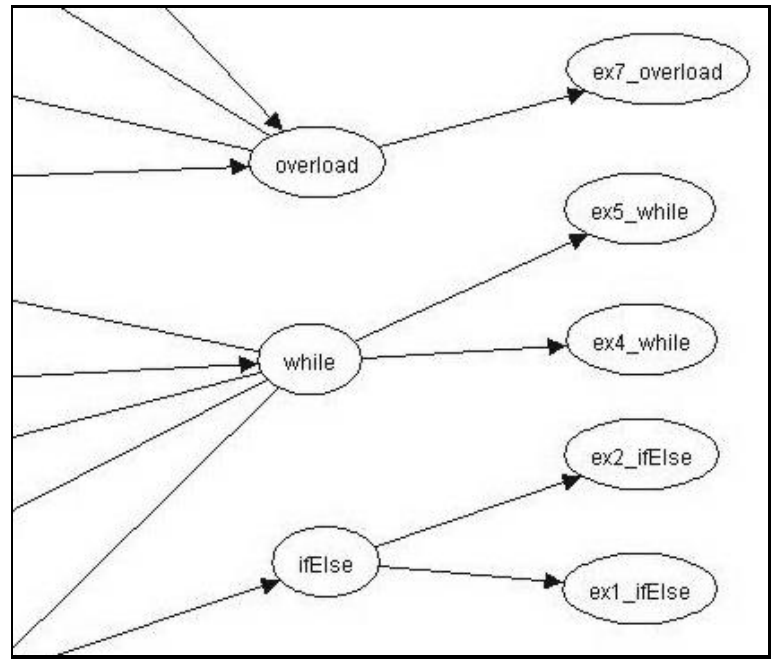

Figura 3: Trecho da Rede Bayesiana com nós de evidência
Para este trabalho, foram utilizados ao todo nove nós de evidência (ver seção 7). No entanto, novos nós podem ser criados e adicionados à rede, o que inclusive aumentaria a capacidade da rede para espelhar de maneira fidedigna o conhecimento atual do aluno.

\section{Avaliação}

Na ausência de um STI que possa utilizar o modelo bayesiano de aluno desenvolvido, o modelo bayesiano proposto não pode passar por uma avaliação com usuários reais. Dessa forma, o método utilizado para avaliar a rede foi de alunos virtuais, que tem sido amplamente utilizado na avaliação de modelos de alunos [12, 47]. Entretanto, a abordagem utilizada se diferiu da mais comumente empregadas na literatura que envolve sistemas especialistas. Geralmente, os alunos virtuais são implementados como um subconjunto de regras de um sistema especialista do domínio em questão, juntamente com algumas regras de falsas concepções. No trabalho proposto, devido à ausência de um sistema especialista, os alunos virtuais são representados por resultados de avaliações com desempenhos variados.

Os nós de evidência foram inseridos na rede com o principal objetivo de permitir um processo de avaliação e validação do modelo de aluno. Estes nós deveriam receber evidências de exercícios específicos: no caso desta avaliação, três conteúdos foram selecionados: while, ifelse, e overload (sobrecarga). Para cada conteúdo, três exercícios diferentes foram incluídos. A ideia principal era testar os resultados que a rede apresentaria para um conjunto de alunos com variados graus de conhecimento nos conteúdos selecionados. A Tabela 2 mostra os resultados desta avaliação fictícia.

De acordo com a Tabela 2, há 30 alunos virtuais que teriam sido submetidos a um teste incluindo 9 questões. Cada um dos alunos teve um desempenho diferente, e nesta tabela foram classificados de acordo (a tabela apresenta os alunos ordenados por desempenho). A tabela superior mostra os acertos e erros dos alunos para cada questão. $\mathrm{Na}$ última linha, é exibida a pontuação total daquele estudante (cada questão vale 1,1). A tabela inferior mostra a pontuação do aluno para os conceitos de ifelse, while e overload. Os valores foram calculados somando todos os acertos do aluno para as questões envolvendo cada conceito. Nesse caso, cada questão foi considerada como valendo 33,3, para que o resultado fosse um valor entre 0 e 100 , como o resultado retornado pela rede.

A Tabela 3 mostra os resultados apresentados pela Rede Bayesiana após ter recebido como evidência os acertos (ou erros) das questões para cada um dos alunos (Tabela 2). Como pode ser observado nessa tabela, a rede retorna um valor entre 0 e $100 \%$ para cada conceito (cada nó da rede), representando a probabilidade do aluno co- 
nhecer o conceito em questão. Embora tenham sido fornecidas evidências apenas para os conceitos de if-else, while e overload, a rede é capaz de inferir o conhecimento do estudante para os outros conceitos devido às relações entre os nós estabelecidas na rede (Figura 2).

Apesar de alguns resultados inesperados, a rede reagiu de forma coerente com os valores inseridos. Por exemplo, o aluno 3 teve um desempenho ótimo nas questões de while, mediano nas questões de if-else, e péssimo nas questões de overload. A rede mostrou coerência nestes conceitos. Entretanto, o percentual atribuído ao if-else foi um pouco acima do esperado. De forma semelhante, $o$ aluno 4 obteve um desempenho mediano nas questões de while e, desta vez, a rede atribuiu um percentual mais adequado a este conceito.

Tabela 2: Resultados de uma prova fictícia para 30 alunos virtuais.

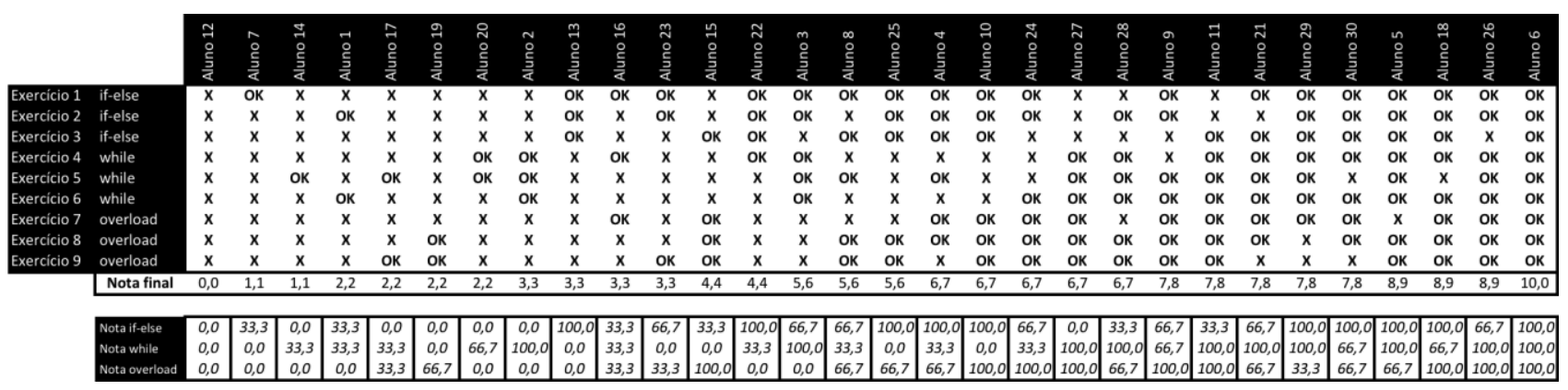

Tabela 3: Resultados da Rede Bayesiana para alguns conceitos de programação.

\begin{tabular}{|c|c|c|c|c|c|c|c|c|c|}
\hline & $\begin{array}{l}\frac{N}{0} \\
\stackrel{\underline{U}}{ \pm}\end{array}$ & $\frac{\frac{\omega}{\frac{T}{3}}}{\frac{2}{3}}$ & के & $\underset{\frac{\pi}{\pi}}{\frac{\vec{t}}{\pi}}$ & $\stackrel{\substack{c \\
\frac{1}{2}}}{\frac{1}{2}}$ & $\begin{array}{l}\stackrel{0}{0} \\
\frac{0}{2} \\
\frac{0}{0} \\
\frac{0}{0} \\
0\end{array}$ & 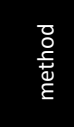 & 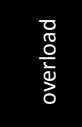 & 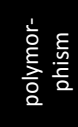 \\
\hline Aluno 1 & 0,02 & 0,02 & 1,00 & 1,10 & 0,04 & 0,06 & 0,06 & 0,00 & 2,15 \\
\hline Aluno 2 & 0,00 & 100,00 & 4,21 & 2,43 & 1,58 & 1,21 & 0,82 & 0,00 & 2,20 \\
\hline Aluno 3 & 99,74 & 100,00 & 33,34 & 18,39 & 19,98 & 14,93 & 9,87 & 0,00 & 2,76 \\
\hline Aluno 4 & 100,00 & 30,86 & 57,83 & 89,34 & 93,43 & 95,02 & 96,63 & 98,33 & 89,88 \\
\hline Aluno 5 & 100,00 & 100,00 & 96,58 & 94,93 & 99,35 & 99,48 & 99,63 & 99,80 & 92,54 \\
\hline Aluno 6 & 100,00 & 100,00 & 96,71 & 95,08 & 99,51 & 99,65 & 99,81 & 100,00 & 92,72 \\
\hline Aluno 7 & 0,02 & 0,00 & 1,00 & 1,10 & 0,04 & 0,06 & 0,06 & 0,00 & 2,15 \\
\hline Aluno 8 & 96,39 & 29,75 & 55,80 & 86,85 & 90,78 & 93,02 & 95,29 & 97,67 & 88,69 \\
\hline Aluno 9 & 99,98 & 99,98 & 96,70 & 95,08 & 99,51 & 99,65 & 99,81 & 100,00 & 92,72 \\
\hline Aluno 10 & 100,00 & 0,00 & 41,47 & 88,86 & 92,91 & 95,21 & 97,54 & 100,00 & 90,75 \\
\hline Aluno 11 & 31,32 & 100,00 & 91,66 & 90,81 & 94,98 & 96,60 & 98,25 & 100,00 & 91,37 \\
\hline Aluno 12 & 0,00 & 0,00 & 1,00 & 1,09 & 0,04 & 0,06 & 0,06 & 0,00 & 2,15 \\
\hline Aluno 13 & 100,00 & 0,00 & 1,67 & 2,43 & 1,58 & 1,21 & 0,85 & 0,00 & 2,20 \\
\hline Aluno 14 & 0,00 & 0,02 & 1,00 & 1,10 & 0,04 & 0,06 & 0,06 & 0,00 & 2,15 \\
\hline Aluno 15 & 0,23 & 0,00 & 1,55 & 25,02 & 25,07 & 49,63 & 74,20 & 100,00 & 70,53 \\
\hline Aluno 16 & 0,02 & 0,02 & 1,00 & 1,11 & 0,05 & 0,08 & 0,09 & 0,04 & 2,18 \\
\hline Aluno 17 & 0,00 & 0,02 & 1,00 & 1,10 & 0,05 & 0,08 & 0,09 & 0,04 & 2,18 \\
\hline Aluno 18 & 100,00 & 99,98 & 96,70 & 95,08 & 99,51 & 99,65 & 99,81 & 100,00 & 92,72 \\
\hline Aluno 19 & 0,00 & 0,00 & 1,37 & 20,14 & 19,96 & 39,68 & 59,39 & 80,09 & 56,88 \\
\hline Aluno 20 & 0,00 & 68,41 & 3,20 & 2,01 & 1,09 & 0,85 & 0,58 & 0,00 & 2,18 \\
\hline Aluno 21 & 99,98 & 100,00 & 96,58 & 94,92 & 99,35 & 99,48 & 99,63 & 99,80 & 92,54 \\
\hline Aluno 22 & 100,00 & 3,71 & 2,84 & 3,02 & 2,26 & 1,72 & 1,15 & 0,00 & 2,22 \\
\hline Aluno 23 & 68,50 & 0,00 & 1,57 & 2,26 & 1,36 & 1,13 & 0,87 & 0,30 & 2,45 \\
\hline Aluno 24 & 97,04 & 30,40 & 57,08 & 88,86 & 92,91 & 95,21 & 97,54 & 100,00 & 90,75 \\
\hline Aluno 25 & 100,00 & 0,00 & 40,55 & 86,85 & 90,78 & 93,02 & 95,29 & 97,67 & 88,69 \\
\hline Aluno 26 & 99,98 & 100,00 & 96,71 & 95,08 & 99,51 & 99,65 & 99,81 & 100,00 & 92,72 \\
\hline Aluno 27 & 0,00 & 100,00 & 89,36 & 88,86 & 92,91 & 95,21 & 97,54 & 100,00 & 90,75 \\
\hline Aluno 28 & 30,86 & 100,00 & 90,22 & 89,34 & 93,43 & 95,02 & 96,63 & 98,33 & 89,88 \\
\hline Aluno 29 & 100,00 & 100,00 & 36,46 & 22,13 & 23,86 & 19,05 & 14,23 & 4,82 & 7,09 \\
\hline Aluno 30 & 100,00 & 99,98 & 96,57 & 94,92 & 99,35 & 99,48 & 99,63 & 99,80 & 92,54 \\
\hline
\end{tabular}


De maneira geral, alguns percentuais atingiram valores acima ou abaixo do previsto. Mesmo assim, não apresentaram resultados absurdos e inversões (conceitos que deveriam apresentar percentual menor do que outros na verdade apresentarem uma porcentagem maior), ainda que algumas relações tenham mostrado alguma proximidade imprevista. Por exemplo, polimorfismo (polymorphism) tem seus valores elevados de forma considerável quando o aluno acerta as questões de sobrecarga (overlo$a d$ ), sendo que nem sempre ambos os conceitos estão tão atrelados.

A Figura 4 ilustra as taxas de diferença entre os resultados das inferências realizadas pela rede com os resultados concretos dos alunos virtuais para os conceitos if-else, while e overload. Como pode ser observado na figura, para a grande maioria dos alunos (em torno de 65\%), a diferença foi menor do que $5 \%$, o que é um valor muito pequeno. Isso significa que a rede consegue inferir tão bem quanto um teste para esses alunos.

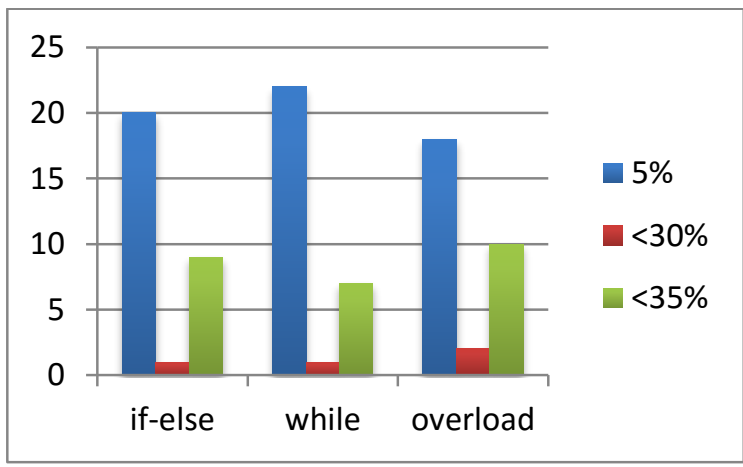

Figura 4: Diferenças entre os resultados da inferência da Rede Bayesiana com os resultados dos alunos virtuais.

Para os alunos restantes (em torno de $35 \%$ ), as diferenças nunca ultrapassaram $35 \%$, o que é um resultado bastante satisfatório. Essa diferença de valores entre o resultado do teste e o inferido pela rede se deve principalmente ao modo como ambos avaliam o conhecimento. Os professores avaliam o conhecimento do aluno em algum conceito se baseando principalmente no resultado das questões que envolvem aqueles conceitos específicos. Por outro lado, a Rede Bayesiana considera igualmente o desempenho do aluno em conceitos pré-requisitos.

\section{Conclusão}

O ensino de programação é onipresente em cursos de Ciência da Computação, e a utilização de ferramentas que contribuam para a plena aprendizagem dos estudantes traz muitos benefícios. Para os professores, inclusive, a disponibilidade de uma ferramenta de suporte poderá significar menos sobrecarga e feedback adequado àqueles que mais necessitam.

STIs são poderosos ambientes computacionais de ensino e aprendizagem. Eles se caracterizam por prover ensino e assistência individualizada aos estudantes. Algumas revisões sistemáticas realizadas nos últimos cinco anos mostraram que STIs podem ser tão efetivos quanto professores particulares [21, 43, 44, 46]. Assim como alunos que tem aulas com um professor particular, alunos que recebem o apoio de um STI tem um desempenho até 2 sigmas maior que alunos que recebem instrução em sala de aula [46].

A efetividade dos STIs como ferramenta educacional se deve principalmente pelo fato de que eles personalizam o conteúdo e assistência ao conhecimento e outras características do aluno. Dessa forma, um componente essencial do STI é o Modelo de Aluno, pois é este que infere e mantem uma base de informações sobre o aluno, o que permite adaptar a instrução. Redes Bayesianas tem sido empregadas na construção de Modelos de Aluno, pois estas são particularmente interessantes para inferência sobre dados incertos, tais como conhecimento e estados afetivos do aprendiz.

Dessa forma, esse artigo apresentou uma proposta de Modelo probabilístico de Aluno para o domínio de programação. A estrutura da rede bem como suas probabilidades foram montadas a partir de entrevistas com especialistas, professores com longa experiência no ensino de programação. A rede proposta foi avaliada com a técnica de alunos virtuais. Através de simulações de 30 alunos virtuais, foi possível verificar que os resultados da rede ficaram muito próximos dos resultados das avaliações, em geral, apresentando uma diferença menor que $5 \%$ ou no máximo de até $35 \%$.

Outros trabalhos em modelos probabilísticos de alunos tem mostrado que essa é uma forma eficiente de inferir o conhecimento do aluno. Entretanto, avaliações com usuários reais se fazem necessárias para mostrar a real efetividade do método para ensino de lógica de programação. Dessa forma, para um trabalho futuro, pretende-se integrar o modelo de aluno proposto a um STI de programação em desenvolvimento pelo grupo e realizar a avaliação da Rede Bayesiana com usuários reais.

\section{Agradecimentos}

Os pesquisadores agradecem aos professores de programação da UNISINOS que auxiliaram na definição dos principais conceitos da Rede Bayesiana, assim como na sua avaliação. 


\section{Referências}

[1] A list of elementary patterns that would be useful for teaching introductory programming: http://max.cs.kzoo.edu/ abrady/patterns/Progra mmingPatternsList.html.

[2] Arroyo, I., Cooper, D., Burleson, W., Woolf, B.P., Muldner, K. and Christopherson, R. 2009. Emotion Sensors Go To School. International Conference on Artificial Intelligence in Education (2009), 17-24.

[3] Arroyo, I., Woolf, B.P., Cooper, D.G., Burleson, W. and Muldner, K. 2011. The Impact of Animated Pedagogical Agents on Girls' and Boys' Emotions, Attitudes, Behaviors and Learning. Advanced Learning Technologies (ICALT), 2011 11th IEEE International Conference on (2011), 506-510.

[4] Baker, R., Corbett, A. and Aleven, V. 2008. More accurate student modeling through contextual estimation of slip and guess probabilities in bayesian knowledge tracing. Intelligent Tutoring Systems. (2008), 406-415.

[5] Baker, R., Walonoski, J., Heffernan, N.T., Corbett, A. and Koedinger, K.R. 2008. Why Students Engage in "Gaming the System" Behavior in Interactive Learning Environments. Journal of Interactive Learning Research. 19, 2 (2008), 185-224.

[6] Bälter, O. and Bailey, D.A. 2010. Enjoying Python, processing, and Java in CS1. ACM Inroads.

[7] Berlin, D.- and Bennedsen, J. 2006. What do Teachers Teach in Introductory Programming ? Proceedings of the Second International Workshop on Computing Education Research (ICER '06) (2006), 17-28.

[8] Bloom, B.S. 1984. The 2 Sigma Problem: The Search for Methods of Group Instruction as Effective as One-to-One Tutoring. Educational Researcher. 13, 6 (Jun. 1984), 4-16.

[9] Brusilovsky, P. 2011. Student model centered architecture for intelligent learning environment. International Conference on User Modeling (2011), 31-36.

[10] Brusilovsky, P. 1994. The Construction and Application of Student Models in Intelligent Tutoring Systems. International Journal of Computer and System Sciences. 32, 1 (1994), 7089.

[11] Butz, C.J., Hua, S. and Maguire, R.B. 2004. A Web-Based Intelligent Tutoring System for Computer Programming. IEEEWICACM International Conference on Web Intelligence WI04. 4, (2004), 159-165.
[12] Collins, J.A., Greer, J. and Huang, S.X. 1996. Adaptive Assessment using Granularity Hierarchies and Bayesian Nets. Third International Conference on Intelligent Tutoring Systems (1996), 569-577.

[13] Conati, C. 2010. Bayesian Student Modeling. Advances in Intelligent Tutoring Systems. 281299.

[14] Conati, C., Gertner, A. and VanLehn, K. 2002. Using Bayesian networks to manage uncertainty in student modeling. User Modeling and UserAdapted Interaction. 12, 4 (2002), 371-417.

[15] Conati, C., Gertner, A., VanLehn, K. and Druzdzel, M.J. 1997. On-line student modeling for coached problem solving using Bayesian networks. User Modeling: Proceedings of the Sixth International Con-ference, UM97. (1997), 231-242.

[16] Deitel, H. and Deitel, P. 2012. Java How to Program. Prentice Hall.

[17] Gertner, A., Conati, C. and VanLehn, K. 1998. Procedural help in Andes: Generating hints using a Bayesian network student. Proceedings of the 15th National Conference on Artificial Intelligence (1998), 106-111.

[18] Horstmann, C.S. 2004. Big Java. Bookman.

[19] Liu, C.L. 2006. Using Bayesian networks for student modeling. Cognitively Informed Systems: Utilizing Practical Approaches to Enrich Information Presentation and Transfer. E.M. Alkhalifa, ed. Idea Group. 282-309.

[20] Liu, C.L. 2005. Using mutual information for adaptive item comparison and student assessment. Educational Technology \& Society. 8, (2005), 100-119.

[21] Ma, W., Adesope, O.O., Nesbit, J.C. and Liu, Q. 2014. Intelligent tutoring systems and learning outcomes: A meta-analysis. Journal of Educational Psychology. 106, 4 (2014), 901-918.

[22] Martin, J. and VanLehn, K. 1995. Student assessment using Bayesian nets. Int. J. of HumanComputer Studies. 42, 6 (1995), 575-591.

[23] McCracken, M., Almstrum, V., Diaz, D., Guzdial, M., Hagan, D., Kolikant, Y.B.-D., Laxer, C., Thomas, L., Utting, I. and Wilusz, T. 2001. A multi-national, multi- institutional study of assessment of programming skills of first-year CS students. ACM SIGCSE Bulletin. 33, (2001), 125-180.

[24] Neapolitan, R.E. 2003. Learning Bayesian Networks. Prentice-Hall.

[25] Nielsen, T.D. and Jensen, F. V 2009. Bayesian Networks and Decision Graphs. Springer.

[26] Nouh, Y., Karthikeyani, P. and Nadarajan, R. 2007. Intelligent Tutoring System-Bayesian Student Model. 2006 1st International 
Conference on Digital Information Management (Apr. 2007), 257-262.

[27] Pearl, J. 1986. A constraint-propagation approach to probabilistic reasoning. Uncertainty in Artificial Intelligence. 357-370.

[28] Pearl, J. 1993. Belief networks revisited. Artificial Intelligence.

[29] Pearl, J. 1988. Probabalistic Reasoning in Intelligent Systems. Morgan Kaufmann.

[30] Pears, A., Seidman, S., Malmi, L., Mannila, L., Adams, E., Bennedsen, J., Devlin, M. and Paterson, J. 2007. A survey of literature on the teaching of introductory programming. $A C M$ SIGCSE Bulletin. 39, 4 (Dec. 2007), 204.

[31] Proulx, V.K. 2000. Programming patterns and design patterns in the introductory computer science course. ACM SIGCSE Bulletin. 32, 1 (Mar. 2000), 80-84.

[32] Reye, J. 2004. Student modelling based on belief networks. International Journal of Artificial Intelligence in Education. 14, (2004), 1-33.

[33] Rogalski, J. and Samurçay, R. 1990. Acquisition of programming knowledge and skills. Psychology of programming. 18, (1990), 157174.

[34] Russell, S.J. and Norvig, P. 2010. Artificial Intelligence: A Modern Approach. Prentice Hall.

[35] Santhi, R., Priya, B. and Nandhini, J.M. 2013. Review of intelligent tutoring systems using bayesian approach. National Conference on "Computational Linguistics and Integrated Classical Knowledge (Feb. 2013).

[36] Seffrin, H., Rubi, G. and Jaques, P.A. 2013. Uma Rede Bayesiana aplicada na identificação do conhecimento algébrico. Simpósio Brasileiro de Informática na Educação (Campinas, 2013).

[37] Self, J.A. 1994. Formal approaches to student modelling. Student Modelling: The Key to Individualized Knowledge-Based Instruction. G.I. Mccalla and J. Greer, eds. Springer. 295-295.

[38] Self, J.A. 1979. Student models and artificial intelligence. Computers \& Education. 3, (1979), 309-312.

[39] Self, J.A. 1999. The defining characteristics of intelligent tutoring systems research: ITSs care, precisely. International Journal of Artificial Intelligence in Education. (1999), 350-364.

[40] Shute, V.J. and Psotka, J. 1996. Intelligent tutoring systems: Past, Present and Future. Handbook of Research on Educational Communications and Technology. Scholastic Publications.

[41] Shute, V.J. and Regian, J.W. 1993. Principles for evaluating intelligent tutoring systems. Journal of Artificial Intelligence in Education. 4, 2-3 (1993), 245-271.
[42] Stajduhar, I. and Dalbelo-Basić, B. 2010. Learning Bayesian networks from survival data using weighting censored instances. Journal of biomedical informatics. 43, 4 (Aug. 2010), 61322.

[43] Steenbergen-Hu, S. and Cooper, H. 2014. A meta-analysis of the effectiveness of intelligent tutoring systems on college students' academic learning. Journal of Educational Psychology. 106, (2014), 331-347.

[44] Steenbergen-Hu, S. and Cooper, H. 2013. A meta-analysis of the effectiveness of intelligent tutoring systems on $\mathrm{K}-12$ students' mathematical learning. Journal of Educational Psychology. 105, (2013), 970-987.

[45] VanLehn, K. 2006. The Behavior of Tutoring Systems. International Journal of Artificial Intelligence in Education. 16, 3 (2006), 227-265.

[46] Vanlehn, K. 2011. The Relative Effectiveness of Human Tutoring, Intelligent Tutoring Systems, and Other Tutoring Systems. Educational Psychologist. 46, 4 (Oct. 2011), 197-221.

[47] VanLehn, K., Niu, Z., Siler, S. and Gertner, A. 1998. Student Modeling from Conventional Test Data: A Bayesian Approach without Priors. Intelligent Tutoring Systems. 434-443.

[48] Vygotsky, L.S. 1978. Mind in Society. Harvard University Press.

[49] Woolf, B.P. 2007. Building Intelligent Interactive Tutors. Morgan Kaufmann.

[50] Zhou, X. and Conati, C. 2003. Inferring user goals from personality and behavior in a causal model of user affect. Proceedings of the 8 th international conference on Intelligent user interfaces (New York, NY, USA, 2003), 211218. 